\title{
Safety and efficacy of renal sympathectomy in ablation of hypertension complicated with atrial fibrillation
}

\author{
Yongzhong Zhang ${ }^{1}$, Lin Zhao ${ }^{1}$, Xiaoshu Yin ${ }^{1}$, Xiaoping Wu ${ }^{2, *}$
}

\author{
${ }^{1}$ Department of Vasculocardiology, \\ Ganzhou City People's Hospital, 341000 \\ Ganzhou, Jiangxi, China \\ ${ }^{2}$ Department of Neurology, Taizhou \\ Central Hospital (Taizhou University \\ Affiliated Hospital), 318000 Taizhou, \\ Zhejiang, China

\section{*Correspondence wuxiaoping_666@163.com (Xiaoping $\mathrm{Wu}$ )}

\begin{abstract}
At present, there is limited data on the effect of renal sympathectomy combined with pulmonary vein isolation on hypertensive patients with atrial fibrillation. The purpose of this study was to explore the efficacy and safety of renal sympathectomy combined with ablation in the treatment of hypertension with atrial fibrillation. A comprehensive literature search was conducted on the treatment of hypertension complicated with atrial fibrillation. Comparing the clinical data of renal sympathetic denervation $(\mathrm{RSDN})+$ pulmonary vein isolation $(\mathrm{PVI})$ with that of $\mathrm{PVI}$ alone, we analyzed recurrence of postoperative atrial fibrillation (AF) as main outcome. Classification variables were represented by risk ratio (RR), continuous variables as average difference (MD) and 95\% confidence interval (CI). A total of 7 eligible studies were included, including 734 patients (RSDN+PVI group: 340 cases; PVI group: 394 cases). The follow-up period was one year or more. Compared with the separate PVI (56\%), the recurrence rate of AF in RSDN+PVI group (32\%) decreased significantly (RR: $0.60,95 \%$ CI: 0.50 , $0.71, p<0.001$ ). RSDN+PVI increased the operation time (Weighted Mean Difference (WMD): $24.92,95 \%$ CI: 20.27, 29.57, $p<0.001$ ), but did not increase the fluoroscopy time (WMD: $1.58,95 \% \mathrm{CI}:-7.50,10.66, p=0.73$ ). Six months after operation, the glomerular filtration rate in RSDN+PVI group was significantly higher than that in PVI group (WMD: 6.17, 95\% CI: 3.09, 9.24, $p<0.001$ ). The control of systolic blood pressure and diastolic blood pressure in RSDN+PVI group was significantly better than that in PVI group (WMD: $-9.55,95 \% \mathrm{CI}:-16.27,-2.84, p=0.005$; WMD: $-6.11,95 \%$ CI: $-10.72,-1.50, p=0.009)$. There was no significant difference in complications between the two groups (RR: $0.85,95 \%$ CI: $0.32,2.21, p=0.73$ ). The heterogeneity of clinical outcome was very low in atrial fibrillation and complications $\left(I^{2}=0\right)$. PVI combined with RSDN is a feasible and safe interventional therapy option for patients with hypertension complicated with atrial fibrillation, which can effectively reduce the burden of atrial fibrillation and improve the control of blood pressure and prevent further renal damage.
\end{abstract}

\section{Keywords}

Hypertensive; Atrial fibrillation; Ablation; Renal sympathectomy

\section{Introduction}

Atrial fibrillation (AF) is the most common arrhythmia in the world, and its incidence in adult population is about $2-3 \%$. The risk of atrial fibrillation and thromboembolism increases with age and hypertension. Moreover, hypertension is also the most common cardiovascular disease that leads to the development and recurrence of atrial fibrillation [1]. Current AF treatment schemes can be divided into drug therapy and/or ablation therapy [2]. Despite the continuous improvement of tools and techniques, many patients still have symptoms, side effects of drug therapy. Meanwhile, the recurrence rate after catheter ablation is high, especially for patients with hypertension [3]. Previous studies have shown that the interaction between hyperactive sympathetic nervous system and hypertension is known to be related to the progression of $\mathrm{AF}$ [4]. In pathophysiology, the disorders of sympathetic and vagal nervous system play an important role in the pathogenesis of hypertension and atrial fibrillation [5]. Although renal sympathetic denervation (RSDN) has been studied to control hypertension and improve atrial fibrillation, its safety and treatment have not been fully evaluated. The purpose of this study was to evaluate the safety and efficacy of renal sympathetic denervation (RDN) in the treatment of hypertension complicated with atrial fibrillation.

\section{Materials and methods}




\subsection{Retrieval strategy}

We search Public Medicine (PubMed), The Cochrane Library, Excerpta Medica Database (EMbase), China National Knowledge Infrastructure (CNKI), Chinese Scientific Journal Database (VIP) and Wanfang databases by the computer, and the search time is limited to September 2020. Chinese key words: hypertension, catheter ablation, atrial fibrillation, renal nerve block, randomized control; English key words: Hypertension, catheter ablation, atrial fibrillation, Renal nerve removal, Radiofrequency catheter ablation (RFCA), AF, randomized controlled trial. We used the above search words as subject words or free words, then the logical operators to formulate the search form, meanwhile, manual retrieval. If you have any questions, contact the author of the newsletter [6]. The searching strategy in the PubMed is listed as the following: (((Hypertension [Title/Abstract])) AND ((catheter ablation [Title/Abstract] OR RFCA [Title/Abstract]) OR (atrial fibrillation [Title/Abstract] OR AF [Title/Abstract]) OR (Renal nerve removal [Title/Abstract]))) AND ((randomized controlled trial [Title/Abstract] OR RCT [Title/Abstract])). All the references were managed by Endnote X8.0 (Clarivate Analytics, Philadelphia, PA, USA).

\subsection{Literature inclusion criteria}

Patients in the literature had a history of symptomatic paroxysmal atrial fibrillation and were scheduled to receive catheter ablation supported by guidelines and had a significant clinical history of hypertension. Systolic blood pressure is greater than or equal to $130 \mathrm{mmHg}$, diastolic blood pressure is greater than or equal to $80 \mathrm{mmHg}$, or both, and taking at least one antihypertensive drug.

The intervention was to perform pulmonary vein isolation catheter ablation combined with renal denervation with the patient's full informed consent, while the control group was treated with isolated pulmonary vein catheter ablation alone. The main outcome is recurrence of atrial fibrillation. The secondary outcome indicators are operation time, fluoroscopy time, postoperative blood pressure and complications. We used the longest follow-up data obtained from individual studies for analysis.

\subsection{Literature exclusion standard}

(1) The diagnostic method is not clear; (2) there are other diseases; (3) the dose and course of treatment of the test group and the control group are not consistent with the abovementioned intervention measures or the description of the treatment method is unknown; (4) the outcome index cannot be counted; (5) non-RCT literature and non-clinical trial studies; (6) literature published repeatedly or with incomplete data; (7) full-text literature is not available.

\subsection{Literature evaluation and data extraction}

Data extraction: two researchers independently retrieve the literature and extract relevant materials. If there are differences during the period, they can be resolved through joint discussion and, if necessary, be assisted by a third researcher. The research-related methodology included quality evaluation, use and utilization.

The "bias risk assessment" tool recommended by Cochrane Handbook 5.0 (Cochrane Collaboration, Oxford, UK) evaluates the quality of the random allocation method, allocation concealment, blind method, integrity of result data and selective reporting bias of the study. For each study, the above six items were evaluated as yes (low bias), no (highly biased) or "unclear" (lack of relevant information or uncertainty of bias) [7].

\subsection{Statistical method}

Use the Rev-Man5.2 version (Cochrane Collaboration, Oxford, UK) of the software provided by Cochrane collaboration network. The odd ratio (OR) was used as the curative effect analysis statistic, and the weighted average difference (WMD) was used as the curative effect analysis statistic. Each effect was expressed by $95 \%$ confidence interval $(95 \% \mathrm{CI}) \cdot \chi^{2}$ test was used to analyze the heterogeneity among the studies. When there was a high degree of statistical heterogeneity among the studies $\left(p<0.1, I^{2}>50 \%\right)$, the random effect model was used, otherwise the fixed effect model was used [8].

\section{Result}

\subsection{Search result}

A total of 632 literatures were detected, including repetitive literatures and reading abstracts and full texts, excluding case reports, reviews, retrospective studies, non-randomized controlled trials, inconsistent trial bases, etc. and finally included 7 literatures. The retrieval process is shown in Fig. 1 .

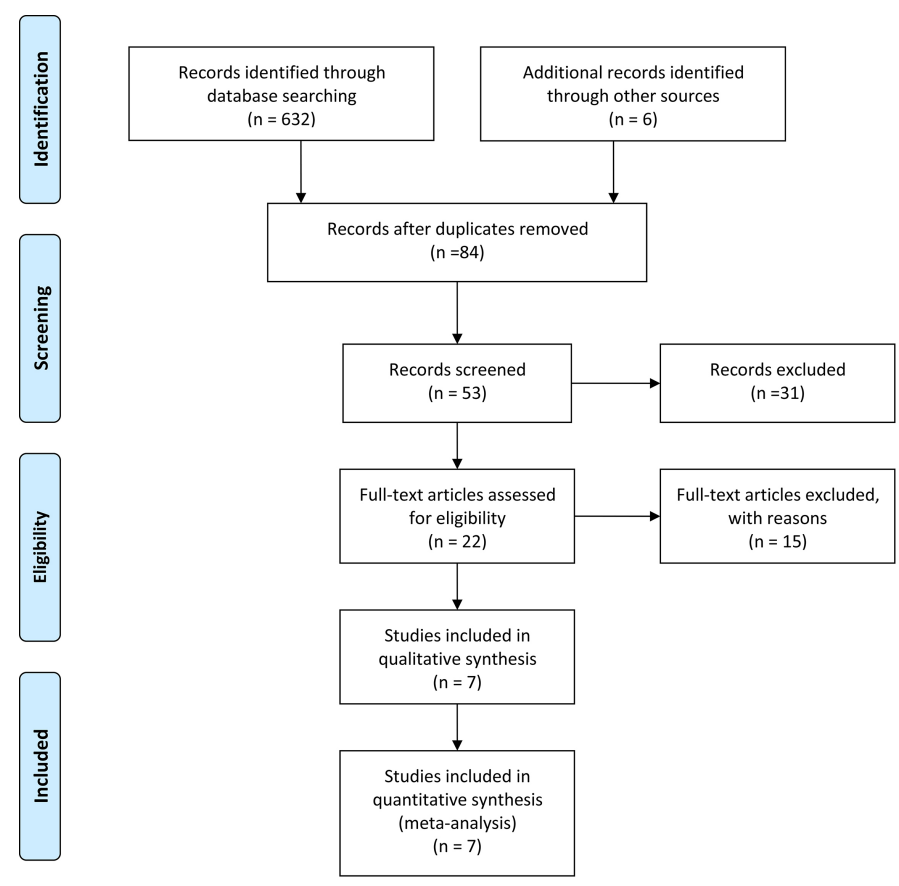

F I G U R E 1. The retrieval process. 
TAB L E 1. Characteristics of included studies.

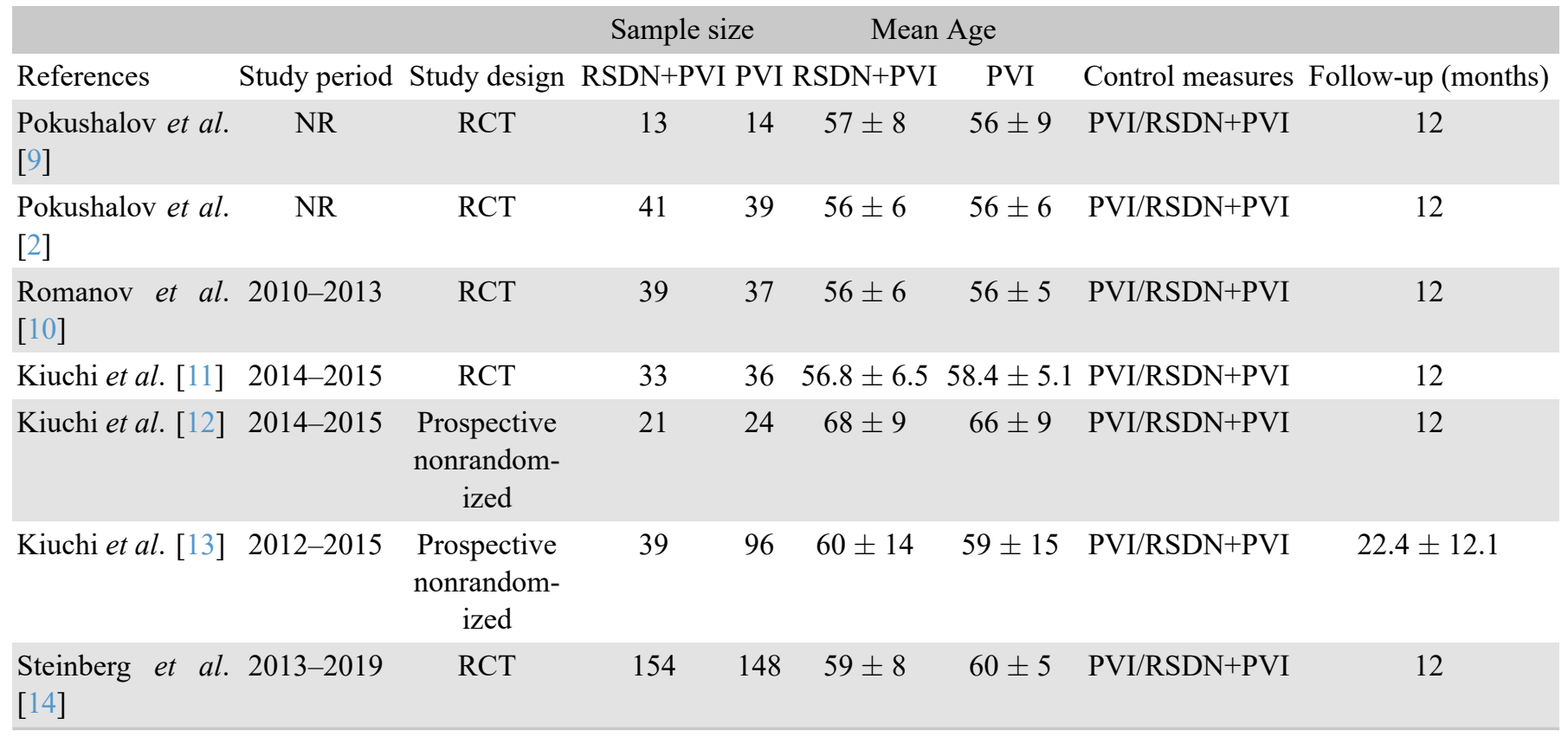

NR: Not reported; RCT: Randomized controlled trial; RSDN: Renal sympathetic denervation; PVI: Pulmonary vein isolation.

TA B L E 2. Quality evaluation.

\begin{tabular}{lcccc} 
Study (year) & Randomization & Double blinding & Withdrawals/dropouts & Jadad score \\
\hline Pokushalov et al. [9] (2012) & Appropriate & Appropriate & Yes & 5 \\
\hline Pokushalov et al. [2] (2014) & Appropriate & Appropriate & Yes & 5 \\
\hline Romanov et al. [10] (2017) & Appropriate & Appropriate & Yes & 5 \\
Kiuchi et al. [11] (2018) & Appropriate & Appropriate & Yes & 5 \\
Kiuchi et al. [12] (2016) & Inappropriate & Appropriate & Yes & 3 \\
Kiuchi et al. [13] (2017) & Inappropriate & Appropriate & Yes & 3 \\
\hline Steinberg et al. [14] (2020) & Appropriate & Inappropriate & Yes & 3 \\
\hline
\end{tabular}

\subsection{General characteristics and quality evaluation of the included study}

Among the 7 articles included, it was mentioned that the baseline of the test group was similar to that of the control group and was comparable (Table 1, Ref. [2, 9-14]) and Fig. 2 (Risk of bias summary). Random words were mentioned in all studies. All the literatures use blind method and distributive concealment. Selective reporting of the findings and other sources of bias are unclear (Table 2, Ref. [2, 9-14]).

\subsection{Meta-analysis results}

\subsubsection{Recurrence of atrial fibrillation}

The heterogeneity test showed that $I^{2}=0 \%$, so the fixed effect model was used. After the combination of all the studies, (RR: $0.60,95 \%$ CI: $0.50,0.71, p<0.001)$, there was a statistically significant difference between the two groups (Fig. 3). It was considered that the AF recurrence rate in the RSDN+PVI group (32\%) was significantly lower than that in the PVI group $(56 \%)$.

\subsubsection{Complications}

The heterogeneity test showed that $I^{2}=0 \%$, so the fixed effect model was used. After the combination of various studies, (RR: $0.85,95 \%$ CI: 0.32, 2.21, $p=0.73$ ), there was no significant difference between the two groups (Fig. 4). Thus it could not be considered that there was a difference in the incidence of complications between the RSDN+PVI group and the PVI group.

\subsubsection{Operation time}

After the heterogeneity test, the outcome showed that $I^{2}=$ $45 \%$, so the fixed effect model was used. After the combination of all the studies, (WMD: 24.92, 95\% CI: 20.27, 29.57, $p<0.001$ ), there was significant difference between the two groups (Fig. 5). It can be considered that the operation time of the RSDN+PVI group is significantly longer than that of PVI alone.

\subsubsection{Perspective time}

After heterogeneity test, $I^{2}=88 \%$, so the random effect model is adopted. After the combination of various studies, (WMD: $1.58,95 \%$, CI: $-7.50,10.66, p=0.73$ ), there was no significant difference between the two groups (Fig. 6). So it cannot be 


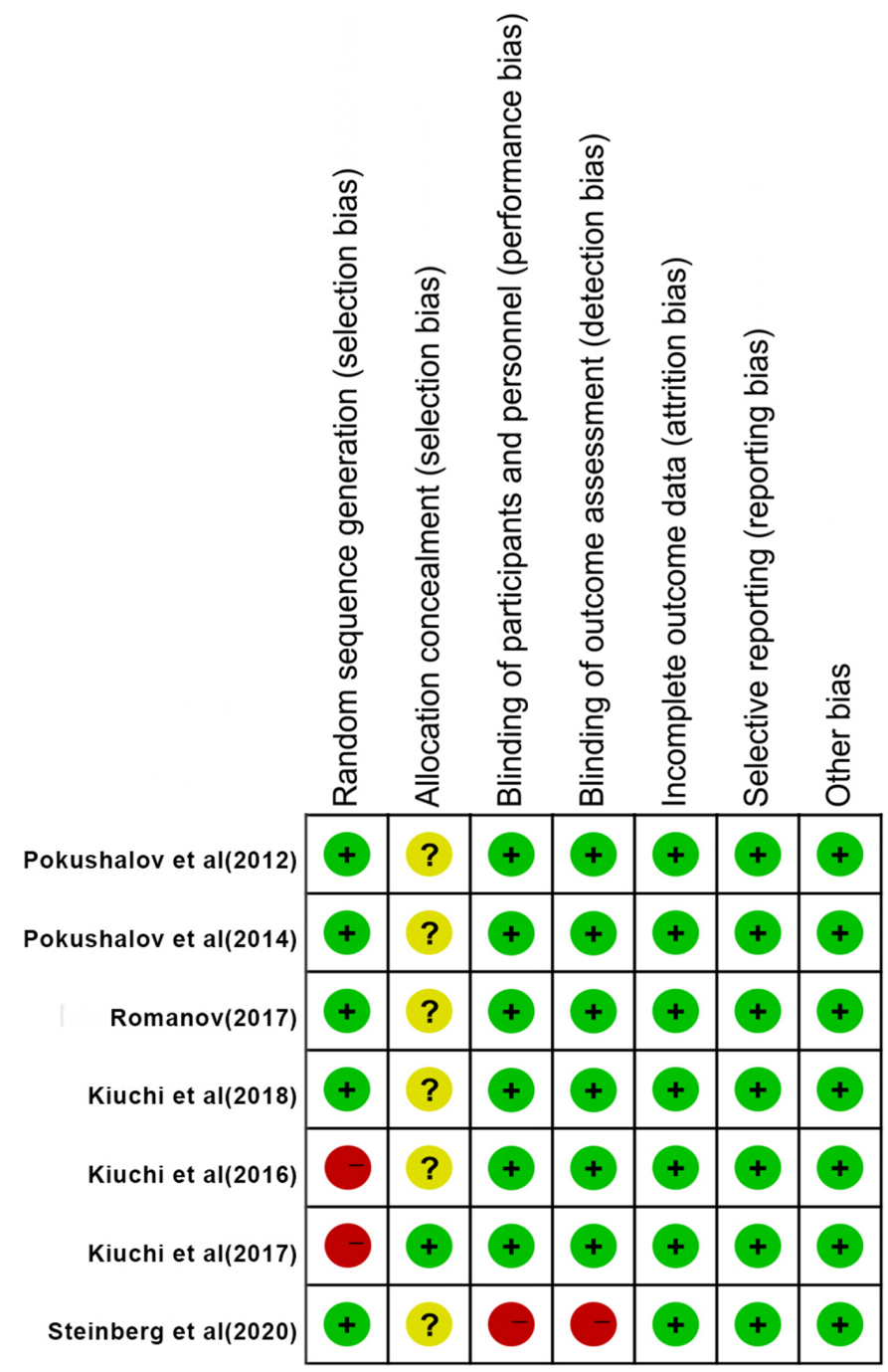

F I G U R E 2. Risk of bias summary. The green circle: Low risk of bias; The yellow circle: unclear risk of bias; The red circle: High risk of bias.

\begin{tabular}{|c|c|c|c|c|c|c|c|c|c|c|c|}
\hline Study or Subgroup & $\begin{array}{l}\text { RSDN+1 } \\
\text { Events }\end{array}$ & $\begin{array}{l}\text { PVI } \\
\text { Total }\end{array}$ & $\begin{array}{r}\text { PVI } \\
\text { Events }\end{array}$ & Total & Weight & $\begin{array}{c}\text { Risk Ratio } \\
\text { M-H, Fixed, 95\% Cl }\end{array}$ & \multicolumn{5}{|c|}{$\begin{array}{c}\text { Risk Ratio } \\
\text { M-H, Fixed, } 95 \% \mathrm{Cl}\end{array}$} \\
\hline Kiuchi 2016 & 5 & 21 & 18 & 24 & $8.7 \%$ & $0.32[0.14,0.71]$ & & & & & \\
\hline Pokushalov 2012 & 4 & 13 & 10 & 14 & $5.0 \%$ & $0.43[0.18,1.04]$ & & & & & \\
\hline Kiuchi 2018 & 13 & 33 & 23 & 36 & $11.3 \%$ & $0.62[0.38,1.01]$ & & & & & \\
\hline Pokushalov 2014 & 15 & 41 & 23 & 39 & $12.2 \%$ & $0.62[0.38,1.00]$ & & & & & \\
\hline Kiuchi 2017 & 15 & 39 & 59 & 96 & $17.6 \%$ & $0.63[0.41,0.96]$ & & & & & \\
\hline Steinberg 2020 & 43 & 154 & 64 & 148 & $33.7 \%$ & $0.65[0.47,0.88]$ & & & & & \\
\hline Romanov 2017 & 15 & 39 & 22 & 37 & $11.6 \%$ & $0.65[0.40,1.04]$ & & & & & \\
\hline Total $(95 \% \mathrm{Cl})$ & & 340 & & 394 & $100.0 \%$ & $0.60[0.50,0.71]$ & & & & & \\
\hline Total events & 110 & & 219 & & & & & & & & \\
\hline $\begin{array}{l}\text { Heterogeneity: } \text { Chi }^{2}= \\
\text { Test for overall effect }\end{array}$ & $\begin{array}{l}36, d f=6 \\
=5.66(P\end{array}$ & $\begin{array}{l}3(P=0 \\
<<0.00\end{array}$ & $\begin{array}{l}.76) ; I^{2}= \\
001)\end{array}$ & & & & 0 & 0. & & 10 & 100 \\
\hline
\end{tabular}

F I G U R E 3. Forest map of recurrent atrial fibrillation between the experimental group and the control group.

considered that the implementation of renal sympathectomy increased the time for patients to receive fluoroscopy.

\subsubsection{Glomerular filtration}

After the heterogeneity test, $I^{2}=52 \%$, so the random effect model was used. After the combination of all the studies, (WMD: 6.17, 95\% CI: 3.09, 9.24, $p<0.001$ ), there was sig- nificant difference between the two groups (Fig. 7). Therefore, 6 months after operation, the glomerular filtration rate in the RSDN+PVI group was significantly higher than that in the PVI group. 


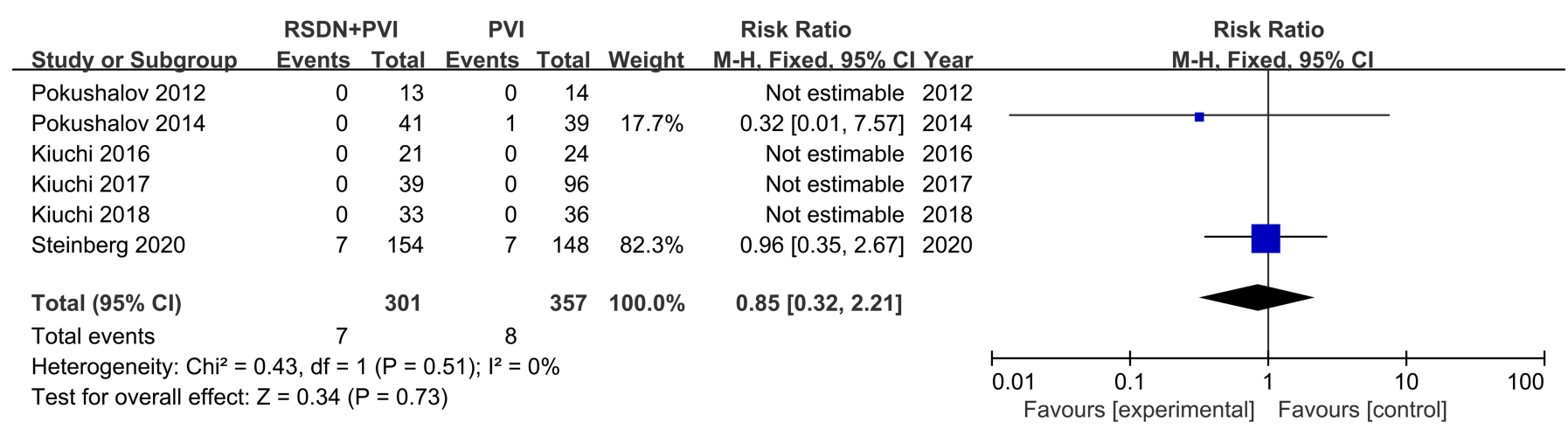

F I G U R E 4. Forest map of complications between the experimental group and the control group.

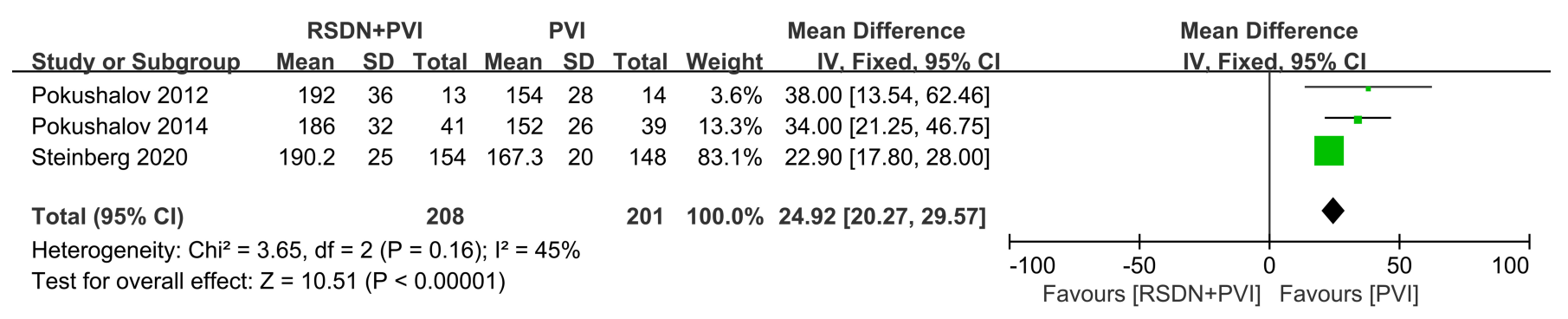

F I G U R E 5. Forest map of operation time between experimental group and control group.

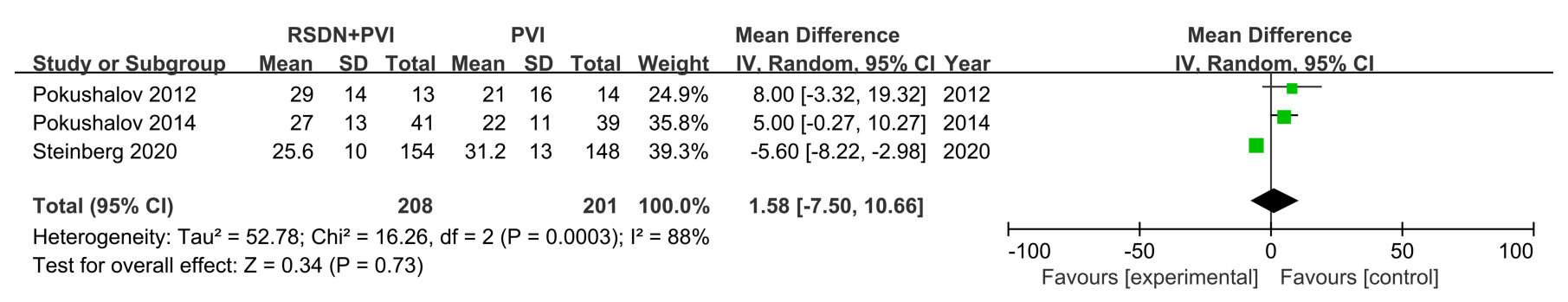

F I G U R E 6. The forest map of perspective time between the experimental group and the control group.

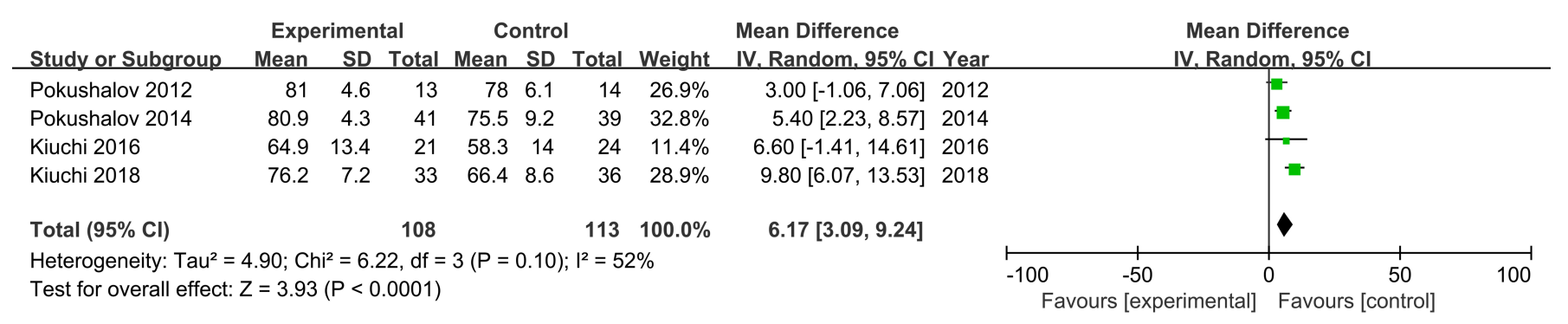

F I G U R E 7. Glomerular filtration rate forest map of experimental group and control group.

\subsubsection{Postoperative blood pressure}

After heterogeneity test, $I^{2}$ is $52 \%$ and $95 \%$ respectively, so both systolic blood pressure and diastolic blood pressure use random effect model, and the differences between the two groups are statistically significant. For the patients in the RSDN+PVI group, the control of systolic blood pressure (WMD: $-9.55,95 \%$ CI: $-16.27,-2.84, p=0.005$ ) (Fig. 8) and diastolic blood pressure (WMD: $-6.11,95 \% \mathrm{CI}:-10.72$, $-1.50, p<0.009$ ) (Fig. 9) was significantly better than that of the PVI group alone.

\section{Discussion}

Hypertension is a major health problem worldwide and is associated with increasing risk of adverse cardiovascular events. On the other hand, atrial fibrillation is the most common arrhythmia, and its prevalence is expected to surge among the world's aging population in the next few years. The two most common cardiovascular diseases in the world [15], hypertension and atrial fibrillation have a causal relationship with each other. Excessive activation of visceral sympathetic nerve is one of the important factors that trigger the development of 


\begin{tabular}{|c|c|c|c|c|c|c|c|c|c|c|c|c|c|c|}
\hline \multirow[b]{2}{*}{ Study or Subgroup } & \multicolumn{3}{|c|}{ RSDN+PVI } & \multicolumn{3}{|c|}{ PVI } & \multicolumn{3}{|c|}{ Mean Difference } & \multirow{2}{*}{\multicolumn{5}{|c|}{$\begin{array}{c}\text { Mean Difference } \\
\text { IV. Random, } 95 \% \mathrm{Cl}\end{array}$}} \\
\hline & Mean & SD & Total & Mean & SD & Total & Weight & IV. Random, $95 \%$ C & I Year & & & & & \\
\hline Pokushalov 2012 & 156 & 7 & 13 & 178 & 8 & 14 & $22.8 \%$ & $-22.00[-27.66,-16.34]$ & 2012 & & -- & & & \\
\hline Kiuchi 2016 & 114 & 7 & 21 & 112 & 8 & 24 & $24.4 \%$ & $2.00[-2.38,6.38]$ & 2016 & & & + & & \\
\hline Kiuchi 2018 & 123 & 4 & 33 & 130 & 6 & 36 & $26.3 \%$ & $-7.00[-9.39,-4.61]$ & 2018 & & $=$ & & & \\
\hline Steinberg 2020 & 135 & 9 & 154 & 147 & 9 & 148 & $26.5 \%$ & $-12.00[-14.03,-9.97]$ & 2020 & & - & & & \\
\hline Total $(95 \% \mathrm{Cl})$ & & & 221 & & & 222 & $100.0 \%$ & $-9.55[-16.27,-2.84]$ & & & & & & \\
\hline $\begin{array}{l}\text { Heterogeneity: } \mathrm{Tau}^{2}= \\
\text { Test for overall effect: }\end{array}$ & $\begin{array}{l}3.13 ; \mathrm{C} \\
=2.79\end{array}$ & $\begin{array}{l}i^{2}= \\
(P=\end{array}$ & $\begin{array}{l}55.20, c \\
0.005)\end{array}$ & $f=3($ & $<0$. & 00001) & $;\left.\right|^{2}=95 \%$ & & & -10 & -50 & 0 & & 100 \\
\hline
\end{tabular}

F I G U R E 8. Forest map of postoperative systolic blood pressure between the experimental group and the control group.

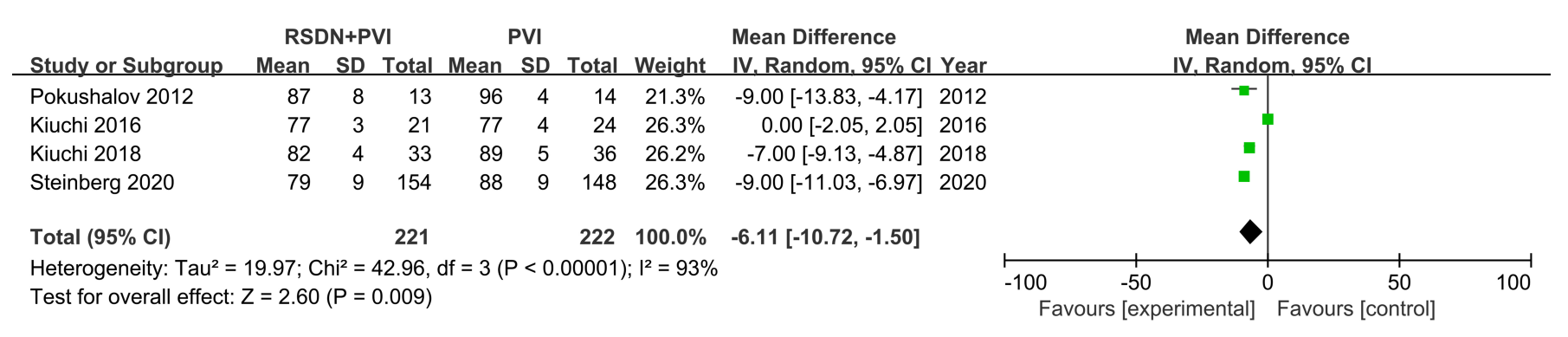

F I G U R E 9. Forest map of postoperative diastolic blood pressure between the experimental group and the control group.

atrial fibrillation. Therefore, controlling blood pressure in an appropriate range can reduce the risk of stroke and cerebral hemorrhage, meanwhile, inhibit the adverse consequences of atrial fibrillation [16]. Because the development of catheter therapy technique, RSDN aims to better control blood pressure and atrial fibrillation by regulating sympathetic nerve activity [10-26].

The main findings of our study included: (1) compared with PVI alone, the recurrence rate of atrial fibrillation in RSDN+PVI group was significantly lower than that in PVI group; (2) the operation time of RSDN+PVI was significantly longer and there was no difference in fluoroscopy time; (3) compared with PVI, RSDN+PVI group, the glomerular filtration rate was significantly higher than that in PVI group; (4) the postoperative systolic and diastolic blood pressure in RSDN+PVI group was significantly lower than that in PVI group. (5) the incidence of complications was low in both groups, and there was no significant difference between the two groups.

In our analysis, we found that only $32 \%$ of patients who received RSDN+PVI had recurrence of atrial fibrillation, compared with $56 \%$ of patients who received PVI alone. Compared with the ablation group, simultaneous renal denervation is more effective in maintaining sinus rhythm (RR: 0.60, 95\% CI: $0.50,0.71, p<0.001)$. It can be considered that compared with PVI alone, RSDN+PVI can reduce the burden of recurrent atrial fibrillation within 12 months by $40 \%$ in patients with refractory paroxysmal atrial fibrillation with a history of hypertension. At the same time, RSDN+PVI can reduce the burden of recurrence of atrial fibrillation within 12 months. RSDN+PVI can effectively alleviate renal damage caused by hypertension and improve glomerular filtration rate $(95 \% \mathrm{CI}$ : $3.09,9.24, p<0.001$ ).

During renal denervation, there is no increased risk of any complications, including renal or renal artery complications. Compared with simple catheter ablation, renal denervation increased the operation time slightly, but did not increase the fluoroscopy time. At the same time, the increase of renal denervation can also significantly improve blood pressure control, reduce the need for cardiovascular hospitalization, and effectively protect kidney function, greatly improving the quality of life of patients.

Finally, our results highlight the clinical benefits and safety of RSDN combined with PVI in patients with paroxysmal atrial fibrillation and hypertension. In this study, 7 clinical trials featuring 734 patients with $\mathrm{AF}$ and hypertension were included. Statistical analysis showed that the homogeneity between the studies was very good during the data merging period. Similarly, the main results were well maintained in random grouping and sensitivity analysis. Therefore, RSDN plus PVI treatment can be used as a better solution for patients with complicated hypertension complicated with atrial fibrillation.

\section{Conclusion}

PVI combined with RSDN may be a feasible and safe interventional therapy for patients with hypertension complicated with atrial fibrillation, which can effectively reduce the burden of atrial fibrillation and reduce blood pressure. Moreover, the combination treatment can improve the control of blood pressure and prevent further kidney damage, providing a synergistic and beneficial effect.

\section{AUTHOR CONTRIBUTIONS}

YZ, LZ - designed the study, supervised the data collection; $\mathrm{XY}$ - analyzed the data, interpreted the data; XW-prepare the manuscript for publication and reviewed the draft of the manuscript; all authors have read and approved the manuscript. 


\section{ETHICS APPROVAL AND CONSENT TO PARTICIPATE}

Not applicable.

\section{ACKNOWLEDGMENT}

Thanks to all the peer reviewers for their opinions and suggestions.

\section{FUNDING}

This research received no external funding.

\section{CONFLICT OF INTEREST}

The authors declare no conflict of interest.

\section{REFERENCES}

[1] Jahangir A, Lee V, Friedman PA, Trusty JM, Hodge DO, Kopecky SL, et al. Long-term progression and outcomes with aging in patients with lone atrial fibrillation: a 30-year follow-up study. Circulation. 2007; 115: 3050-3056.

[2] Pokushalov E, Romanov A, Katritsis DG, Artyomenko S, Bayramova S, Losik D, et al. Renal denervation for improving outcomes of catheter ablation in patients with atrial fibrillation and hypertension: early experience. Heart Rhythm. 2014; 11: 1131-1138.

[3] Mahfoud F, Schmieder RE, Azizi M, Pathak A, Sievert H, Tsioufis $\mathrm{C}$, et al. Proceedings from the 2nd European Clinical Consensus Conference for device-based therapies for hypertension: state of the art and considerations for the future. European Heart Journal. 2017; 38: 3272-3281.

[4] Linz D, Ukena C, Mahfoud F, Neuberger HR, Böhm M. Atrial autonomic innervation: a target for interventional antiarrhythmic therapy? Journal of the American College of Cardiology. 2014; 63: 215-224.

[5] Chen PS, Chen LS, Fishbein MC, Lin SF, Nattel S. Role of the autonomic nervous system in atrial fibrillation: pathophysiology and therapy. Circulation Research. 2014; 114: 1500-1515.

[6] Menzies D. Systematic reviews and meta-analyses. The International Journal of Tuberculosis and Lung Disease. 2011; 15: 582-593.

[7] Moher D, Liberati A, Tetzlaff J, Altman DG. Preferred reporting items for systematic reviews and meta-analyses: the PRISMA statement. PLos Medicine. 2009; 6: e1000097.

[8] Higgins JP, Thompson SG, Deeks JJ, Altman DG. Measuring inconsistency in meta-analyses. The BMJ. 2003; 327: 557-560.

[9] Pokushalov E, Romanov A, Corbucci G, Artyomenko S, Baranova V, Turov A, et al. A randomized comparison of pulmonary vein isolation with versus without concomitant renal artery denervation in patients with refractory symptomatic atrial fibrillation and resistant hypertension. Journal of the American College of Cardiology. 2012; 60: 1163-1170.

[10] Romanov A, Pokushalov E, Ponomarev D, Strelnikov A, Shabanov V, Losik D, et al. Pulmonary vein isolation with concomitant renal artery denervation is associated with reduction in both arterial blood pressure and atrial fibrillation burden: Data from implantable cardiac monitor. Cardiovascular Therapeutics. 2017; 35: e12264.

[11] Kiuchi MG, Chen S, Hoye NA, Pürerfellner H. Pulmonary vein isolation combined with spironolactone or renal sympathetic denervation in patients with chronic kidney disease, uncontrolled hypertension, paroxysmal atrial fibrillation, and a pacemaker. Journal of Interventional Cardiac Electrophysiology. 2018; 51: 51-59.

[12] Kiuchi MG, Chen S, E Silva GR, Paz LM, Kiuchi T, de Paula Filho AG, et al. Pulmonary vein isolation alone and combined with renal sympathetic denervation in chronic kidney disease patients with refractory atrial fibrillation. Kidney Research and Clinical Practice. 2016; 35: 237-244.

[13] Kiuchi MG, Chen S, E Silva GR, Rodrigues Paz LM, Kiuchi T, de Paula Filho AG, et al. The addition of renal sympathetic denervation to pulmonary vein isolation reduces recurrence of paroxysmal atrial fibrillation in chronic kidney disease patients. Journal Of Interventional Cardiac Electrophysiology. 2017; 48: 215-222.

[14] Steinberg JS, Shabanov V, Ponomarev D, Losik, D, Ivanickiy, E, Kropotkin E, et al. Effect of Renal Denervation and Catheter Ablation vs Catheter Ablation Alone on Atrial Fibrillation Recurrence Among Patients With Paroxysmal Atrial Fibrillation and Hypertension: The ERADICATE-AF Randomized Clinical Trial. JAMA. 2020; 323: 248 255.

[15] Kallistratos MS, Poulimenos LE, Manolis AJ. Atrial fibrillation and arterial hypertension. Pharmacological Research. 2018; 128: 322-326.

[16] Kirchhof P, Benussi S, Kotecha D, Ahlsson A, Atar D, Casadei B, et al. 2016 ESC Guidelines for the management of atrial fibrillation developed in collaboration with EACTS. Europace. 2016; 18: 1609-1678.

[17] Gulati R, Raphael CE, Negoita M, Pocock SJ, Gersh BJ. The rise, fall, and possible resurrection of renal denervation. Nature Reviews Cardiology. 2016; 13: 238-244.

[18] Chen S, Kiuchi MG, Acou WJ, Derndorfer M, Wang J, Li R, et al. Feasibility of catheter ablation renal denervation in "mild" resistant hypertension. The Journal of Clinical Hypertension. 2017; 19: 361-368.

[19] Chen S, Kiuchi MG, Schmidt B, Hoye NA, Acou WJ, Liu S, et al. Renal denervation for mild-moderate treatment-resistant hypertension: a timely intervention? Herz. 2019; 44: 412-418.

[20] Kiuchi MG, Mion D, Graciano ML, de Queiroz Carreira MA, Kiuchi T, Chen S, et al. Proof of concept study: improvement of echocardiographic parameters after renal sympathetic denervation in CKD refractory hypertensive patients. International Journal of Cardiology. 2016; 207: 612.

[21] Kiuchi MG, Graciano ML, Carreira MA, Kiuchi T, Chen S, Lugon JR. Long-Term effects of renal sympathetic denervation on hypertensive patients with mild to moderate chronic kidney disease. Journal of Clinical Hypertension. 2016; 18: 190-196.

[22] Kiuchi MG, Graciano ML, de Queiroz Carreira MA, Kiuchi T, Chen $\mathrm{S}$, Andrea BR, et al. Effects of renal sympathetic denervation in left ventricular hypertrophy in CKD refractory hypertensive patients. International Journal of Cardiology. 2016; 202: 121-123.

[23] Wang Z, Chen S, Zhou T, Su L, Ling Z, Fan J, et al. Comparison of saline-irrigated catheter vs. temperature-controlled catheter for renal denervation in a canine model. American Journal of Hypertension. 2015; 28: 1434-1443.

[24] Kiuchi MG, Chen S, Andrea BR, Kiuchi T, Carreira MA, Graciano ML, et al. Renal sympathetic denervation in patients with hypertension and chronic kidney disease: does improvement in renal function follow blood pressure control? Journal of Clinical Hypertension. 2014; 16: 794-800.

${ }^{[25]}$ Lu J, Ling Z, Chen W, Du H, Xu Y, Fan J, et al. Effects of renal sympathetic denervation using saline-irrigated radiofrequency ablation catheter on the activity of the renin-angiotensin system and endothelin-1. Journal of the Renin-Angiotensin-Aldosterone System. 2014; 15: 532539.

[26] Kiuchi MG, Maia GL, de Queiroz Carreira MA, Kiuchi T, Chen S, Andrea BR, et al. Effects of renal denervation with a standard irrigated cardiac ablation catheter on blood pressure and renal function in patients with chronic kidney disease and resistant hypertension. European Heart Journal. 2013; 34: 2114-2121.

How to cite this article: Yongzhong Zhang, Lin Zhao, Xiaoshu Yin, Xiaoping Wu. Safety and efficacy of renal sympathectomy in ablation of hypertension complicated with atrial fibrillation. Signa Vitae. 2022; 18(2): 130-136. doi:10.22514/sv.2022.015. 